\title{
Frequency Analysis of the First and Second Heart Sounds of Humans
}

\author{
Koon-Ong Lim, Ph.D.,* D.R. Boughner, M.D., Ph.D., ${ }^{+}$ \\ Ping W. Hur, Ph.D., ${ }^{+}$and Martin LeE, M.Sc. ${ }^{+}$
}

\begin{abstract}
SUMmary
Each sound signal of the first and the second heart sounds of humans was divided into three equal segments. The frequency spectrum of each segment was analyzed using the fast-Fourier-transform technique. Theory predicts that if the first and second heart sounds are caused principally by the respective vibrations of the atrioventricular and semilunar valves, then the frequency of these sounds should increase with an increase in the pressure difference across these valves. In other words, the frequency of the heart sounds should increase with time, as the pressure difference across these valves increases with time for most of the duration of the sounds. Our frequency analysis, however, showed that there is no systematic relation between the predominant frequency of successive segments of the sound signals. This therefore appears to indicatc that it is quite unlikely that the vibrations of the heart valves are the principal contributor to the origin of the first and second heart sounds.
\end{abstract}

\section{Additional Indexing Words:}

Fast-Fourier-transform

$\mathrm{T}$ HOUGH the presence of the first and second heart sounds in humans has been known for about 360 years, there is to this day, no general consensus regarding the actual cause or causes of these sounds.

Presently there are two divergent views regarding the origin of these two sounds. One view contends that these sounds are produced by the acceleration and deceleration of blood which cause the walls of the heart and the major blood vessels to vibrate ${ }^{1), 2)}$ while a second group of investigators are of the opinion that the sounds are duc to the vibrations of the closed atrioventricular and semilunar valves. ${ }^{3), 4)}$

From the *School of Physics, Universiti Sains Malaysia, Penang, Malaysia and the Departments of Biophysics and Medicine, University of Western Ontario, London, Ontario, Canada.

Address for reprint: Dr. K.O. Lim, School of Physics, Universiti Sains Malaysia, Penang, Malaysia.

Received for publication December 5, 1982.

Manuscript revised June 28, 1983. 
A way to help support or refute the validity of either of these two views is to look at the physics of the vibrating structures that are supposed to be producing these sounds. As far as we are aware, little work has been done in this area. MacCanon et al ${ }^{5}$ considered the energy content of the first heart sound and concluded that the observed motion of the mitral valve is too small to account for the total sound energy measured. Recently, Lim et $\mathbf{a l}^{6}$ ) derived theoretical formulae for the frequencies that would be generated if the tissues of the mitral and aortic valves were considered as vibrating membranes. Their analysis showed that if indeed the first and second sounds are due to the vibrations of the valves, then their frequencies should increase with an increase in the pressure difference across these structures. Their work, therefore, suggests that the role of the valves in the production of the first and second sounds would be better understood if data on the frequency of these sounds as a function of the pressure difference across the valves is known. To our knowledge such data are not available. The aim of this paper is to report the results of our preliminary investigations in this area.

Since the pressure difference across both the atrioventricular and semilunar valves increases with time for most of the duration of the first and second heart sounds, an investigation of their frequency dependence with time should provide us with some information regarding their dependence on pressure difference across the valves. To do this heart sounds from volunteers were recorded. Each sound signal was then divided into 3 equal segments and each segment was then analyzed for its frequency spectrum using the fast-Fourier-transform technique.

\section{MEthods}

The first and second heart sounds from 5 normal, healthy individuals (ages 22 to 33 years, 2 males, 3 females) with no history of cardiovascular disorders were recorded using an echocardiograph recorder (Echo IV by Electronics for Medicine) with a phonocardiograph amplifier (Model M2109) and a microphone placed at the fourth intercostal space of the precordium. A filter which gave the system a 30 to $800 \mathrm{~Hz}$ response was used. Outside the bandwidth, attenuation of frequencies occurred. All recordings were done in a quiet room with the volunteer in the supine position. For reference purposes, the electrocardiogram was also recorded simultaneously.

The technique adopted for the analysis of the sound signals requires that the analogue signals be digitised. In order to enhance the accuracy of the digitisation procedure each recorded sound signal was first enlarged 5 times and the amplified signals were then digitised at a rate of 725 points per second 
using a Hewlett-Packard digitiser (9864 A) and computer (HP 9830). The digitised data were stored in tapes for later use.

To study the characteristics of the sound signals as a function of time, three separate signals of each heart sound were analyzed for each individual and a mean signal was then generated. Each of these mean signals was then divided into three equal segments with each segment being analyzed individually. The fast-Fourier-transform technique was used in the analysis and the frequency spectrum in the form of a spectral density function (SDF) versus frequency was determined and plotted for each segment of each mean sound signal. These were done using a computer.

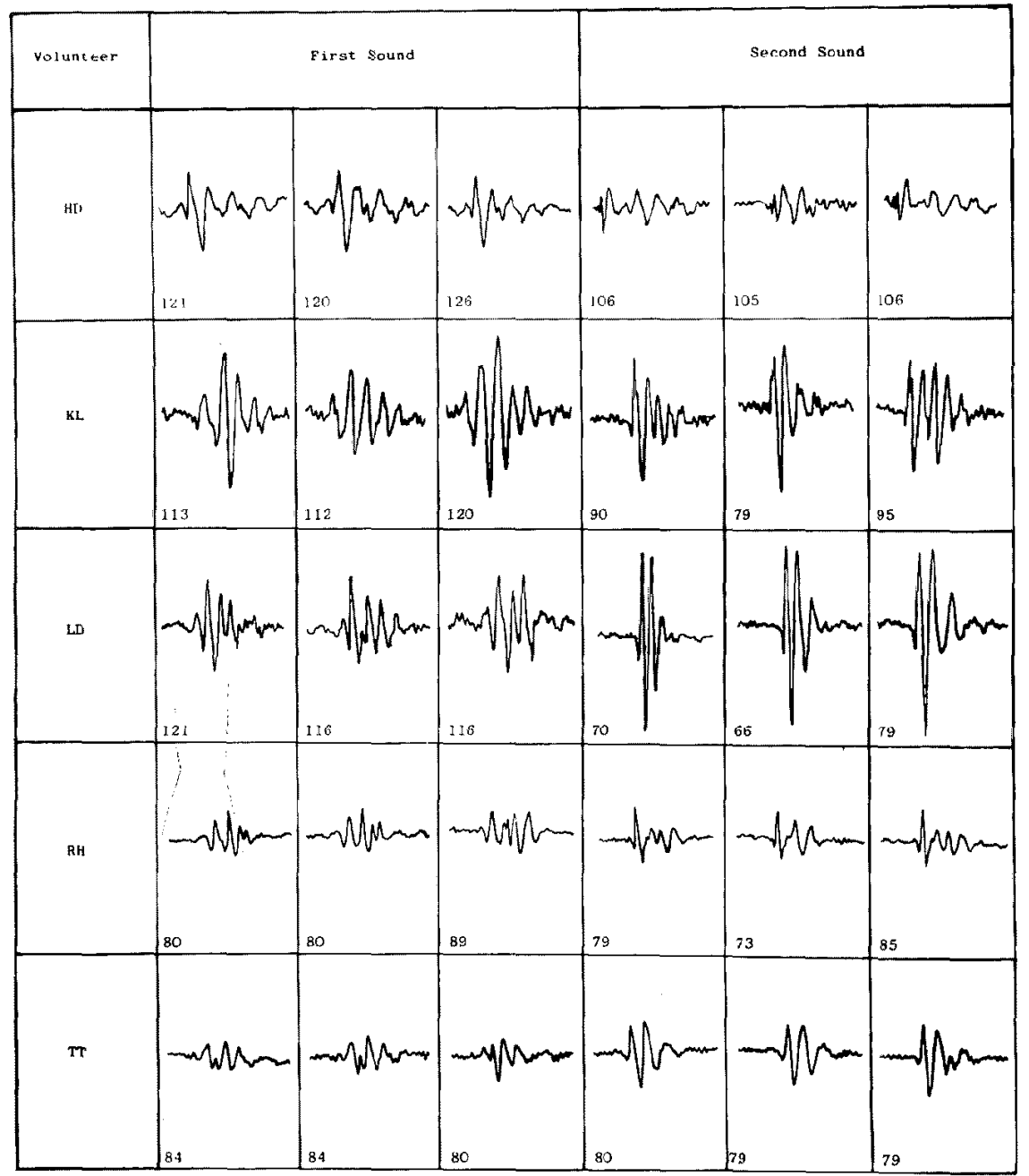

Fig. 1. The original heart sound signals used in the analysis. The numbers at the lower left of each signal denote the duration of the sound in milliseconds. 


\section{RESULTS}

Fig. 1 shows the actual signals that were used in the analysis. The number at the lower left end of each signal denotes the duration (in milliseconds) of each of the signals that were analyzed.

Representative frequency spectra for each of the three segments of the first heart sound are as shown in Fig. 2 while those for the second heart sound are as shown in Fig. 3. The data in Figs. 2 and 3, which are from volunteer TT, show that the frequency spectrum for each segment of the sound contains one major frequency component and that is the frequency component which has the largest value in the spectral density function. This major frequency component is therefore the predominant frequency found within the segment of the sound signal. Similar observations were found in the spectral density function versus frequency plots of other individuals.

Table I lists the predominant frequency found within each segment of each sound signal for each of the 5 volunteers. Since these frequency com-

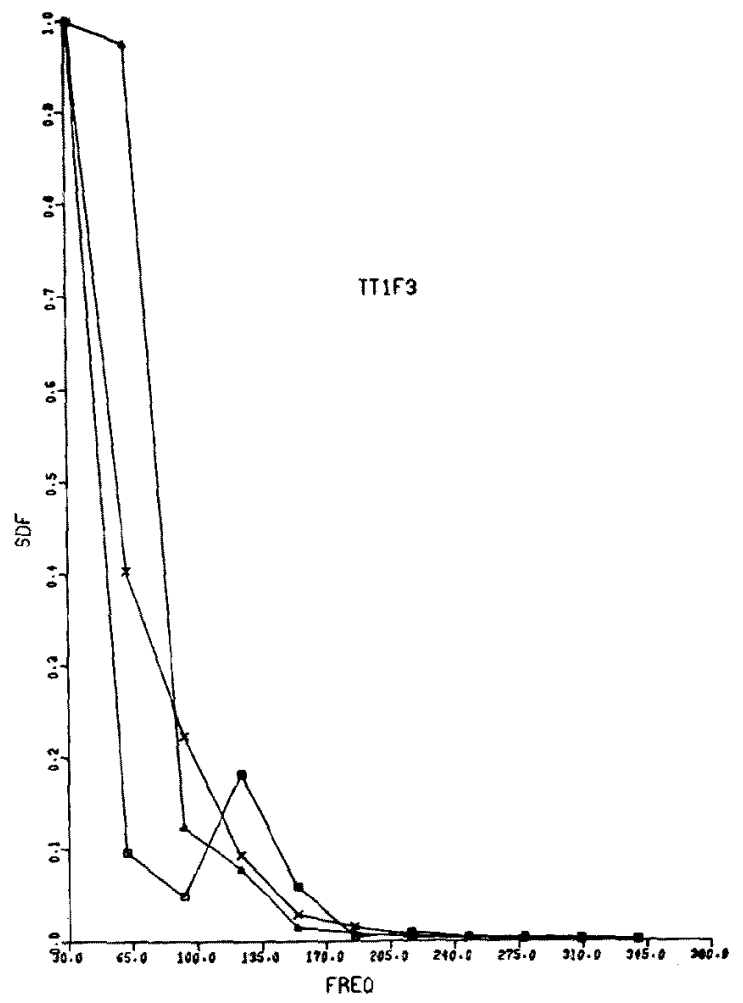

Fig. 2. Frequency spectrum of the three segments of the first heart sound of volunteer TT. $\times$ : first segment, $\triangle$ : second segment, $\square$ : third segment, $\mathrm{SDF}=$ spectral density function; FREQ $=$ sound frequency. 


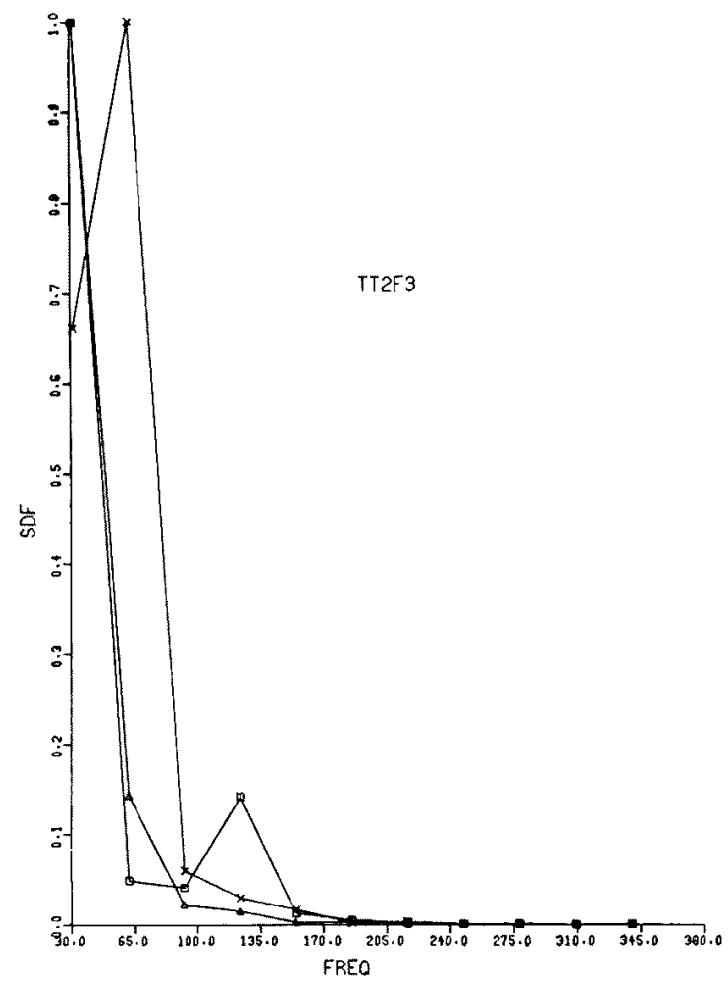

Fig. 3. Frequency spectrum of the three segments of the second heart sound of volunteer TT. $x$ : first segment, $\triangle$ : second segment, $\square$ : third segment.

Table I. Predominant Frequencies of the First and Second Sounds

\begin{tabular}{l|ccc|ccc}
\hline \multirow{2}{*}{ Volunteer } & \multicolumn{3}{|c|}{ First Sound } & \multicolumn{3}{c}{ Second Sound } \\
\cline { 2 - 6 } & $\begin{array}{c}\text { First } \\
\text { segment }\end{array}$ & $\begin{array}{c}\text { Second } \\
\text { segment }\end{array}$ & $\begin{array}{c}\text { Third } \\
\text { segment }\end{array}$ & $\begin{array}{c}\text { First } \\
\text { segment }\end{array}$ & $\begin{array}{c}\text { Second } \\
\text { segment }\end{array}$ & $\begin{array}{c}\text { Third } \\
\text { segment }\end{array}$ \\
\hline HD & 21.9 & 43.9 & 43.9 & 64.8 & 64.8 & 32.4 \\
KL & 24.3 & 48.6 & 48.6 & 68.0 & 68.0 & 34.0 \\
LD & 48.6 & 48.6 & 24.3 & 40.0 & 40.0 & 40.0 \\
RH & 64.8 & 64.8 & 32.4 & 68.0 & 34.0 & 34.0 \\
TT & 30.9 & 30.9 & 30.9 & 61.8 & 30.9 & 30.9
\end{tabular}

Each entry in the table corresponds to the predominant frequency $(\mathrm{Hz})$ found in successive segments of both the first and second heart sounds of 5 individuals.

ponents are those that contain the greatest amount of sound energy within their respective segments, they should therefore correspond to the major frequency components of the vibrating heart valve tissues if indeed these are the structures that produce the heart sounds.

For the first heart sound, the results in Table I show that the predominant 
frequency either increases and then stays constant in the second and third segments or stays constant for the first and second segments and then drops or alternatively stays constant throughout the whole duration of the heart sound. As for the second heart sound, the predominant frequency either stays constant for the first two segments and then decreases or decreases and then remains constant in the second and third segments or alternatively remains constant for all three segments.

These results therefore show that the predominant frequency components of the heart sounds do not appear to exhibit a systematic pattern of either increasing with time, remaining constant with time or decreasing with time. However the results in Table I indicate that the frequency values of the second heart sound are generally higher than those of the first sound.

\section{Discussion}

In recording the sound signals for analysis, a 30 to $800 \mathrm{~Hz}$ system was used. It would have been more desirable if a system which would have allowed passage of lower frequencies had been used instead. However, due to the limitations of the equipment, we had to make do with what was available to us then. In spite of this apparent limitation, we are of the opinion that the heart sound signals recorded are still very meaningful. This is because it has been reported that most of the energy of heart sounds lies above $50 \mathrm{~Hz} .^{7}$ )

Frequency analysis of human heart sounds is not a new endeavour; neither is the use of the fast-Fourier-transform algorithm for their analysis, In fact Yoganathan et $\mathbf{a l}^{8 /, 9}$ have applied the technique for analyzing the frequencies of the first and second heart sounds. They reported that the frequency spectrum for the sound signal, as a whole, exhibited peaks, and that the sound intensity decreased with an increase in frequency. The analysis presented in this paper, however, differs from the work of Yoganathan et $a^{81,93}$ in that we have divided each sound signal into segments and have analyzed the frequency spectrum of each of these segments. We believe that this is the first time that such an analysis has been made. The reason for our doing it has already been mentioned earlier. Our aim would have been better served if each sound signal had been divided into more segments. However, in our analysis we have chosen instead to divide each sound signal into only three equal parts. This is because as the number of segments increases, the accuracy of the analysis decreases and becomes meaningless when too small a segment is used.

Our results in Table I show that the predominant frequency in successive segments of each sound signal does not appear to follow any specific pattern. 
It certainly does not increase with time. Therefore it is unlikely that the frequency of heart sounds increases with the increase in the pressure difference across the valve tissues. Thus the results presented in this study indicate that it is unlikely that the vibrations of the heart valves are the principal contributor to the origin of the first and second heart sounds in man.

\section{ACKNOWLEDGMENTS}

This work was made possible by a Visiting Scientist Award given by the Ontario Heart Foundation to Dr. K.O. Lim.

\section{REFERENGES}

1. Rushmer RF: Cardiovascular Dynamics, 3rd Edition, WB Saunders, Philadelphia, Chapt 10,1970

2. Luisada AA, MacCanon DM, Kumar S, Feigen LP: Changing views on the mechanism of the first and second heart sounds. Am Heart J 88: 503, 1974

3. Leatham A: Splitting of the first and second heart sounds. Lancet 2: 607, 1954

4. Sabbah HN, Stein PD: Investigation of the theory and mechanism of the origin of the second heart sound. Circulat Res 39: 874, 1976

5. MacCanon DM, Bruce DW, Lynch PR, Nickerson JL: Mass excursion parameters of the first sound energy. J Appl Physiol 27: 649, 1969

6. Lim KO, Liew YC, Oh CH: Analysis of mitral and aortic valve vibrations and their role in the production of the first and second heart sounds. Phys Med Biol 25: 727, 1980

7. McKusick VA: Cardiovascular Sound in Health and Disease, Williams \& Wilkins, Baltimore, 1958

8. Joganathan AP, Gupta R, Udwadia FE, Miller JW, Corocoran WH: Use of the fast Fourier transform for frequency analysis of the first heart sound in normal man. Med Biol Eng 14: 69,1976

9. Yoganathan AP, Gupta R, Corocoran WH, Sarma R, Bing RJ, Udwadia FE: Use of the fast Fourier transform in the frequency analysis of the second heart sound in normal man. Med Biol Eng 14: 455, 1976 